\title{
Efficient DS-UWB MUD Algorithm Using Code Mapping and RVM
}

\author{
Pingyan Shi, ${ }^{1}$ Xiaohui Liu, ${ }^{2,3}$ Xiaofeng Tao, ${ }^{1}$ and Jianchao Ji ${ }^{1}$ \\ ${ }^{1}$ China Academy of Space Technology, Xian Branch, Xi'an 710100, China \\ ${ }^{2}$ School of Electronics and Information Engineering, Harbin Institute of Technology, Harbin 150001, China \\ ${ }^{3}$ Institute of Telecommunication Satellite, China Academy of Space Technology, Beijing 100000, China
}

Correspondence should be addressed to Xiaohui Liu; lxhhgd@163.com

Received 3 March 2015; Revised 14 December 2015; Accepted 21 December 2015

Academic Editor: Marzio Pennisi

Copyright (C) 2016 Pingyan Shi et al. This is an open access article distributed under the Creative Commons Attribution License, which permits unrestricted use, distribution, and reproduction in any medium, provided the original work is properly cited.

\begin{abstract}
A hybrid multiuser detection (MUD) using code mapping and a wrong code recognition based on relevance vector machine (RVM) for direct sequence ultra wide band (DS-UWB) system is developed to cope with the multiple access interference (MAI) and the computational efficiency. A new MAI suppression mechanism is studied in the following steps: firstly, code mapping, an optimal decision function, is constructed and the output candidate code of the matched filter is mapped to a feature space by the function. In the feature space, simulation results show that the error codes caused by MAI and the single user mapped codes can be classified by a threshold which is related to SNR of the receiver. Then, on the base of code mapping, use RVM to distinguish the wrong codes from the right ones and finally correct them. Compared with the traditional MUD approaches, the proposed method can considerably improve the bit error ratio (BER) performance due to its special MAI suppression mechanism. Simulation results also show that the proposed method can approximately achieve the BER performance of optimal multiuser detection (OMD) and the computational complexity approximately equals the matched filter. Moreover, the proposed method is less sensitive to the number of users.
\end{abstract}

\section{Introduction}

Ultra wide band (UWB) is an attractive wireless communication technology for its characteristics of high data transmission rate, low power density, high interference resistance, strong multipath resolution, and so on [1-3]. The application range of UWB has been broadened to Wireless Local Area Networks (WLAN), Wireless Sensor Networks (WSN), radar detection, and high-speed communications of indoor and outdoor applications [4-6].

There are mainly two standard schemes of UWB formulated by IEEE $802.15 .3 \mathrm{a}$, that is, the multiband-based orthogonal frequency division multiplexing (MB-OFDM) and impulse-radio-based direct sequence UWB (DS-UWB) [7]. Compared with MB-OFDM scheme, DS-UWB has many attractive advantages such as low peak-to-average power ratio, significant ability of information hidden, and less sensitivity to multipath fading $[8,9]$. In a sense, the multiple access scheme of DS-UWB is similar to code division multiple access (CDMA) systems; both of them use pseudorandom (PN) codes to distinguish different users. However, as in conventional CDMA systems, DS-UWB systems also suffer from the multiple access interference (MAI). The optimal multiuser detector (OMD) proposed by Verdu [10] could achieve the single user's BER performance, but it had a very high computational complexity and was too expensive to handle [11]. Therefore, suboptimal detectors which may approximate OMD's BER performance with an acceptable computational complexity have become a focus of research. In recent years, artificial swarm algorithms are widely used in multiuser detection (MUD). Literature [12] investigates an efficient multiuser detector by selection of initial states based on code mapping for the artificial bee colony algorithm. A complexity-performance-balanced MUD method based on artificial fish swarm algorithm for DS-UWB is introduced in [13]; the BER performance of these methods can approximate the performance of OMD while these artificial swarm algorithms need iterated operation. 
A multiuser frequency-domain turbo detector was employed which combines FD turbo equalization schemes with soft interference cancellation [14]. A code-aided interference suppression method was introduced for narrow band interference restriction in DS-UWB systems [15]. Adaptive MUD methods using the recursive least square (RLS) principles were studied in $[16,17]$. In [18], a low-complexity approximate SISO MUD using soft interference cancellation and linear minimum mean square error (MMSE) filtering for coded CDMA system was introduced. However, few studies were reported that can approximate OMD's BER performance with a computational complexity approximates to linear MUD.

In this paper, a novel MUD algorithm which combines a code mapping method and a wrong code recognition based on RVM is proposed to achieve a BER performance approximate to OMD method with a very low complexity which almost equals the complexity of matched filter.

The remainder of this paper is organized as follows. In Section 2, the DS-UWB system models are introduced. And in Section 3, the principles of the proposed algorithm are described, respectively. In Section 4, simulation results that compare the performance of the proposed algorithm and other approaches are illustrated and discussed, followed by conclusions given in Section 5.

\section{System Models}

Consider a $K$-user asynchronous DS-UWB system in additive white Gaussian noise (AWGN) channel, and assume that each user employs the binary phase-shift key (BPSK) modulation. In this paper, Scholtz's monocycle is used as the UWB pulse waveform, which is approximated to the second derivation of Gaussian pulse. And the expression of Scholtz's monocycle is [19]

$$
p(t)=A\left[1-4 \pi\left(\frac{t-t_{d}}{\tau_{m}}\right)^{2}\right] \exp \left[-2 \pi\left(\frac{t-t_{d}}{\tau_{m}}\right)^{2}\right],
$$

where $t_{d}$ and $\tau_{m}$ are the pulse center and the pulse shape parameter, respectively. At the transmitter $k(k=1,2, \ldots, K)$, BPSK symbols $b_{k}(i) \in\{-1,1\}_{i=1}^{M}$ are spread with the specific PN codes $c_{k}(t)$, which are the binary bit stream valued only by -1 or 1 , and $M$ is the length of bits per packet. The symbol duration is denoted by $T_{s}$. We consider that each BPSK symbol can be divided into $N_{c}$ chips each with duration $T_{c}$, where $N_{c}$ equals $T_{s} / T_{c}$. In each chip, a monocycle $p(t)$ is transmitted with the duration of $T_{p}$ to represent the sign of the chip. Practically, the duration of a chip is much longer than the duration of a UWB pulse; that is, $T_{c} \gg T_{p}$. The $k$ th user's transmission signal can be written as

$$
\begin{gathered}
x_{k}(t)=\sum_{i=1}^{M} \sum_{j=0}^{N_{c}-1} b_{k}(i) c_{k}\left(t-(i-1) T_{s}\right) \\
\cdot p\left(t-(i-1) T_{s}-j T_{c}-\tau_{k}\right),
\end{gathered}
$$

where $\tau_{k}$ represents the random delay of the $k$ th transmitter's monocycle, $0 \leq \tau_{k}<T_{c}$.
Assume that each transmitter uses a time-invariant multipath channel in the same band. Let $h(t)$ represent the impulse response of the transmission channel. Furthermore, suppose that UWB signals reach the receiving antenna by $L$ different paths. The channel impulse response can be written as [20]

$$
h(t)=\sum_{l=1}^{L} \alpha_{l} \delta\left(t-\tau_{l}\right)
$$

The received signal that is transmitted from the $k$ th user is given by

$$
v_{k}(t)=x_{k}(t) * h(t)
$$

where $*$ denotes convolution operator.

The total received signal can be written as

$$
r(t)=v(t)+n(t)=\sum_{k=1}^{K} x_{k}(t) * h(t)+n(t),
$$

where $n(t)$ is zero-mean additive white Gaussian noise (AWGN) with the unilateral power spectral density of $N_{0}$.

In the receiver, the traditional receiver of a DS-UWB system consists of a pulse demodulator and a set of matched filters corresponding to each user. Regard signal $r(t)$ as the input of the group of matched filters. Furthermore, the inter symbol interference (ISI) can be ignored when the base-band signal transmission rate is much lower than the UWB pulse rate.

Let vector $\mathbf{y}=\left[y_{1}, y_{2}, \ldots, y_{K}\right]^{T}$ represent the output of the matched filter group, and let vector $\mathbf{b}=\left[b_{1}, b_{2}, \ldots, b_{K}\right]^{T}$ represent the output of sign detectors, so the output of the matched filters can be represented as follows:

$$
\begin{aligned}
& \mathbf{y}=\mathbf{R A d}+\mathbf{n}, \\
& \mathbf{b}=\operatorname{sgn}(\mathbf{y}),
\end{aligned}
$$

where the vector $\mathbf{d}=\left[d_{1}, d_{2}, \ldots, d_{K}\right]^{T}$ denotes the correct bits of each user and the vector $\mathbf{n}=\left[n_{1}, n_{2}, \ldots, n_{K}\right]^{T}$ denotes the output of the AWGN from each user's corresponding matched filter, while the random variable $n_{k}$ is normally distributed and $E\left[n_{k}\right]=0$ and $D\left[n_{k}\right]=N_{0} N_{c} / 2$, $\mathbf{R}=\left(r_{i j}\right)_{K \times K}$ denotes the cross-correlation matrix, where $r_{i j}=\sum_{l=0}^{N_{c}-1} c_{i}(l) c_{j}(l)$ (what is more, $r_{i i}=N_{c}$ ) and $\mathbf{A}=$ $\operatorname{diag}\left(A_{1}, A_{2}, \ldots, A_{K}\right)$, in which the diagonal element $A_{k}(k \in$ $[1, K], k \in N)$ represents the signal amplitude of the $k$ th user.

\section{The Proposed Hybrid Multiuser Detection}

The main purpose of the proposed method is to pick out the error codes among the received signals and correct them. Firstly, the received codes are mapped into a feature space to make the error codes and the right ones different in some properties which can be easily distinguished. Secondly, identify and pick out the wrong codes by using some approaches in the feature space. Figure 1 shows the diagram of the algorithm. The first stage is corresponding to the 


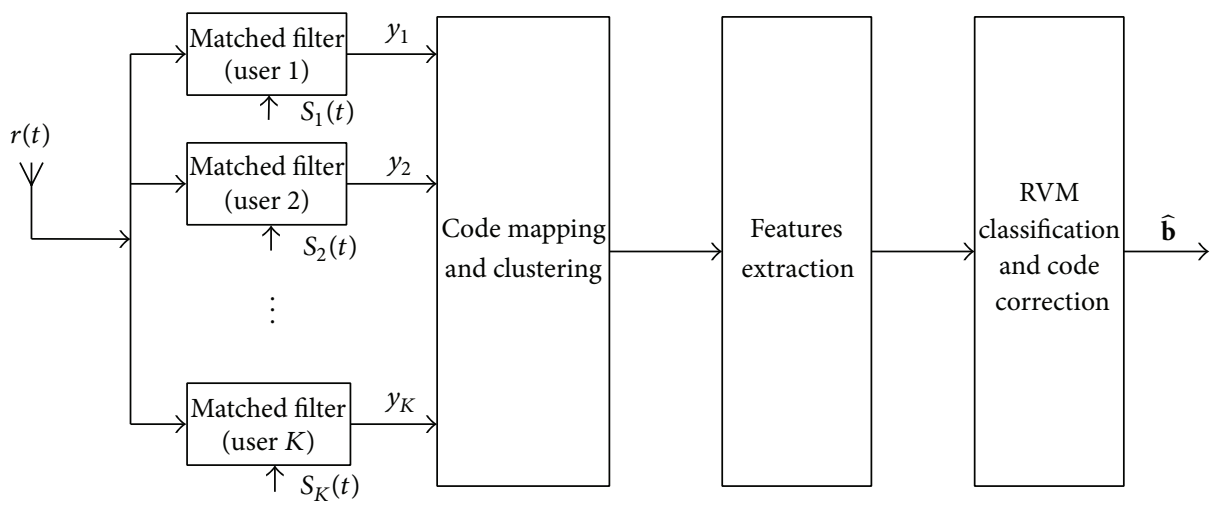

FIgURE 1: The block diagram of the receiver.

code mapping, the second stage is corresponding to features extraction for RVM, and the third stage is corresponding to classification of the codes based on RVM and correcting the wrong codes.

3.1. Code Mapping. Here, a mapping function is constructed to map the output codes set of the matched filter to a onedimensional feature space [21].

According to the theory of optimal multiuser detection described in [22], optimal multiuser detection computes the likehood function

$$
\begin{aligned}
\Lambda(\mathbf{b})= & \int_{0}^{T}\left(r(t)-\sum_{k=1}^{K} A_{k} b_{k} c_{k}(t)\right)^{2} \mathrm{~d} t \\
= & \int_{0}^{T} r(t)^{2} \mathrm{~d} t+\int_{0}^{T}\left(\sum_{k=1}^{K} A_{k} b_{k} c_{k}(t)\right)^{2} \mathrm{~d} t \\
& -\int_{0}^{T} 2 r(t)\left(\sum_{k=1}^{K} A_{k} b_{k} c_{k}(t)\right) \mathrm{d} t
\end{aligned}
$$

and selects the sequence $\left\{b_{k}, 1 \leq k \leq K\right\}$ that minimizes $\Lambda(\mathbf{b})$. Thus the output of OMD can be expressed as

$$
\widehat{\mathbf{b}}_{\mathrm{OMD}}=\arg \left\{\max _{b \in\{-1,1\}}\left(2 \mathbf{b}^{T} \mathbf{A y}-\mathbf{b}^{T} \mathbf{A R A b}\right)\right\} .
$$

It can be easily seen that (9) needs $2^{K}$ trials to get the expected $\mathbf{b}$. The computational complexity of OMD is too high. Thus other methods need to be explored to suppress the multiple access interference. Let

$$
F(\mathbf{b})=\frac{1}{2} \mathbf{b}^{T} \mathbf{A R A} \mathbf{b}-\mathbf{b}^{T} \mathbf{A y} .
$$

According to (9), it can be seen that when the value of function $F(\mathbf{b})$ achieves the minimum, the optimal $\mathbf{b}$ can be obtained. By expanding (10), we get

$$
\begin{aligned}
F(\mathbf{b}) & =F\left(b_{1}, b_{2}, \ldots, b_{K}\right) \\
& =\frac{1}{2} \sum_{i=1}^{K} \sum_{j=1}^{K} A_{i} A_{j} r_{i j} b_{i} b_{j}-\sum_{i=1}^{K} b_{i} A_{i} y_{i} .
\end{aligned}
$$

From (10) and (11), it is clear that function $F(\mathbf{b})$ is a quadratic form of vector $\mathbf{b}$, which means this is a nonlinear equation with the order of two. And it is not easy to solve the minimum of $F(\mathbf{b})$ and the corresponding value of $\mathbf{b}$. In addition, the function $F(\mathbf{b})$ embraces cross-component $b_{i} b_{j}(i, j=1,2, \ldots, K, i \neq j)$, and we cannot judge a code independently by $F(\mathbf{b})$. Hence, it is not appropriate for function $F(\mathbf{b})$ to be the mapping function.

In this case, it is better to calculate the derivative of function $F(\mathbf{b})$ to decrease the order of mapping function. Making the partial derivation of (10), we get

$$
\frac{\partial F}{\partial \mathbf{b}}=\mathbf{A R A b}-\mathbf{A y} .
$$

By expanding (12), we get $K$ th-order linear equations given as follows:

$$
\begin{gathered}
\frac{\partial F}{\partial b_{1}}=\sum_{j=1}^{K} A_{1} A_{j} r_{1 j} b_{j}-A_{1} y_{1} \\
\frac{\partial F}{\partial b_{2}}=\sum_{j=1}^{K} A_{2} A_{j} r_{2 j} b_{j}-A_{2} y_{2} \\
\vdots \\
\frac{\partial F}{\partial b_{K}}=\sum_{j=1}^{K} A_{K} A_{j} r_{K j} b_{j}-A_{K} y_{K} .
\end{gathered}
$$

Because $F(\mathbf{b})$ is a discrete equation, we cannot just set the partial derivative described above equal to 0 and get the stationary point of $F(\mathbf{b})$. Let $L\left(b_{i}\right)=\sum_{j=1}^{K} A_{i} A_{j} r_{i j} b_{j}-A_{i} y_{i}$, $i=1,2, \ldots, K$. Substituting the candidate sequence $\mathbf{b}$ into (13) (the output of matched filter as shown in (7) or results of other conventional suboptimal multiuser detectors can be employed as the candidate sequence $\mathbf{b}$ ), there will be two situations as discussed below.

(1) No Wrong Code in $\mathbf{b}$. Based on the theory of extreme value, if MAI is the only interference resource without AWGN and the elements in $\mathbf{b}$ are all correct, the result of (13) strictly 
equals 0 . And in the condition of high SNR, substituting (6) into $L\left(b_{k}\right)$, we can see that $L\left(b_{k}\right)=-A_{k} n_{k} \sim N\left(0, A_{k}^{2} N_{c} N_{0} / 2\right)$ $(k=1,2, \ldots, K)$ when there is no error bit in the candidate codes set $\mathbf{b}$.

(2) Wrong Codes Exist in $\mathbf{b}$. Suppose that $b_{i}(i \in[1, K], i \in N)$ is the wrong code (in other words, $-b_{i}$ is the correct code); the other codes are correct. Substituting it to the $i$ th equation of (13), we get

$$
\begin{aligned}
L\left(b_{i}\right) & =\sum_{\substack{j=1 \\
j \neq i}}^{K} A_{i} A_{j} r_{i j} b_{j}-A_{i} y_{i}+A_{i}^{2} r_{i i} b_{i} \\
& =\sum_{\substack{j=1 \\
j \neq i}}^{K} A_{i} A_{j} r_{i j} b_{j}-A_{i} y_{i}+A_{i}^{2} r_{i i}\left(-b_{i}\right)+2 A_{i}^{2} r_{i i} b_{i} \\
& =2 A_{i}^{2} N_{c} b_{i}-A_{i} n_{i} .
\end{aligned}
$$

As for $k$ which is not equal to $i$, we get

$$
L\left(b_{k}\right)=2 A_{i} A_{k} r_{i k} b_{i}-A_{k} n_{k}, \quad k=1,2, \ldots, K, \quad k \neq i .
$$

According to (14) and (15), we can see when the $i$ th user's code is wrong; then we get $\left|L\left(b_{i}\right)\right| \gg\left|L\left(b_{k}\right)\right|, k=1,2, \ldots, K, k \neq i$. Therefore, the function $L(\mathbf{b})$ can obviously differentiate the wrong codes and the right codes through the absolute value of it. In addition, $L(\mathbf{b})$ is $K$ th-order linear equations which can get the result of $L(\mathbf{b})$ without complex computations. In conclusion, it is appropriate to set $|L(\mathbf{b})|$ as the optimal decision function in the hybrid MUD algorithm.

It is accessible to map $\mathbf{b}$ into a one-dimension feature space $|L(\mathbf{b})|$ to identify the wrong codes in the candidate set. Figure 2 shows an example of the feature space mapping in the scenario of 10 users, with $6 \mathrm{~dB}$ signal-to-noise ratio (SNR). $|L(\mathbf{b})|$ of 3200 codes are shown in this figure (candidate $\mathbf{b}$ is selected from the result of matched filter as in (7)).

According to Figure 2, it is clear that the wrong codes and the right ones have a significant difference in the feature space mapped by $|L(\mathbf{b})|$.

\subsection{Code Classification and Correction}

3.2.1. Theory of RVM. Tipping proposed the relevance vector machine (RVM) in [23], a sparse Bayesian modeling approach, which enables sparse classification and regression functions to be obtained by linearly weighting a small number of fixed basis functions from a large dictionary of potential candidates. And a significant advantage to support vector machine is that the kernel function of RVM avoids satisfying Mercer's condition [24-26].

In classification, the output function $y(x)$ is defined by

$$
y(x, \boldsymbol{\omega})=\sigma\left(\boldsymbol{\omega}^{T} \phi(x)\right)
$$

where $\sigma(z)=1 /\left(1+e^{-z}\right)$ and $\boldsymbol{\omega}$ denotes the weight matrix.

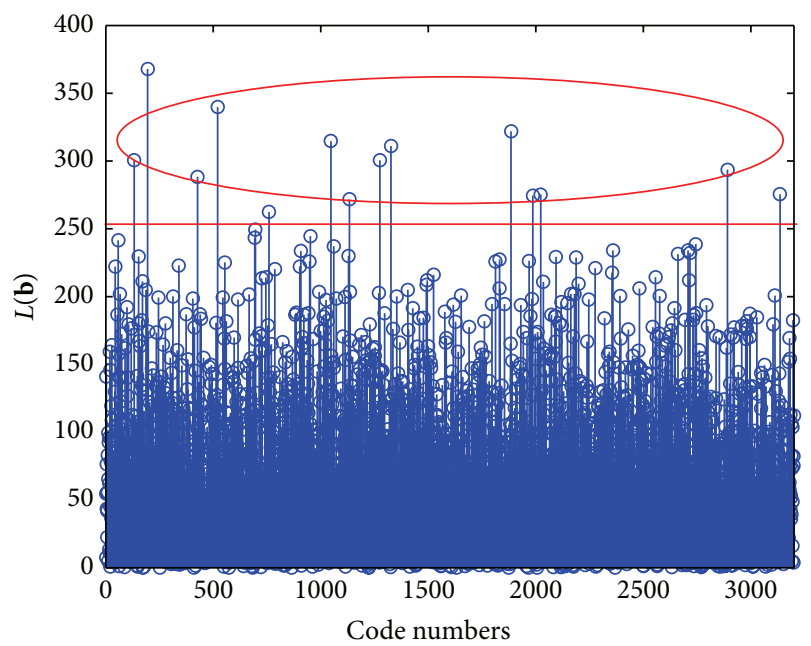

FIGURE 2: Relationship between code numbers and mapping function $\left|L\left(b_{k}\right)\right|$.

Suppose $\boldsymbol{\omega}$ is a Gaussian conditional probability [27], with the 0 expectation and variance $\alpha_{i}^{-1}$. For two classes' classification, the likelihood function is defined by

$$
P(\mathbf{t} \mid \boldsymbol{\omega})=\prod_{n-1}^{N} \sigma\left\{y\left(x_{n}, \boldsymbol{\omega}\right)\right\}^{t_{n}}\left[1-\sigma\left\{y\left(x_{n}, \boldsymbol{\omega}\right)\right\}\right]^{1-t_{n}},
$$

where $t_{n} \in(0,1)$ denote the target value.

Seeking the maximum posterior probability estimation is equivalent to seeking the mode point of the Gaussian function, namely, $\mu_{\mathrm{MP}}$.

Due to the fact that

$$
P(\boldsymbol{\omega} \mid \mathbf{t}, \alpha)=\frac{P(\mathbf{t} \mid \boldsymbol{\omega}) P(\boldsymbol{\omega} \mid \alpha)}{P(\mathbf{t} \mid \alpha)},
$$

the maximum posterior probability estimation according to $\boldsymbol{\omega}$ is equivalent to maximize

$$
\begin{aligned}
\log \{P(\boldsymbol{\omega} \mid \mathbf{t}, \alpha)\}= & \log \{P(\mathbf{t} \mid \boldsymbol{\omega})\}+\log \{P(\boldsymbol{\omega} \mid \alpha)\} \\
& -\log \{P(\mathbf{t} \mid \alpha)\} \\
= & \sum_{n=1}^{N}\left[t_{n} \log y_{n}+\left(1-t_{n}\right) \log \left(-y_{n}\right)\right] \\
& -\frac{1}{2} \boldsymbol{\omega}^{T} \mathbf{A} \boldsymbol{\omega}+C,
\end{aligned}
$$

where $y_{n}=\sigma\left\{y\left(x_{n}, \boldsymbol{\omega}\right)\right\}$ and $C$ denotes a constant. Similarly, the marginal likelihood function can be given by

$$
\begin{aligned}
P(\boldsymbol{\omega} \mid \mathbf{t}, \alpha)= & \int P(\mathbf{t} \mid \boldsymbol{\omega}) P(\boldsymbol{\omega} \mid \alpha) \mathrm{d} \boldsymbol{\omega} P\left(\mathbf{t} \mid \boldsymbol{\omega}_{\mathrm{MP}}\right) \\
& \cdot P\left(\boldsymbol{\omega}_{\mathrm{MP}} \mid \alpha\right)(2 \pi)^{M / 2}|\Sigma|^{1 / 2} .
\end{aligned}
$$

Suppose $\widehat{\mathbf{t}}=\Phi_{\boldsymbol{\omega}_{\mathrm{MP}}}+\mathbf{B}^{-1}(\mathbf{t}-\mathbf{y})$, the approximation of the Gaussian posterior distribution; that is, $\mu_{\mathrm{MP}}=\Sigma \Phi^{T} \mathbf{B} \widehat{\mathbf{t}}$, 
with the variance $\Sigma=\left(\Phi^{T} \mathbf{B} \Phi+\mathbf{A}\right)^{-1}$. The logarithm of the approximate marginal likelihood function is given by

$$
\log P(\mathbf{t} \mid \alpha)=-\frac{1}{2}\left\{N \log (2 \pi)+\log |\mathbf{C}|+\widehat{\mathbf{t}}^{T} \mathbf{C}^{-1} \widehat{\mathbf{t}}\right\},
$$

where $\mathbf{C}=\mathbf{B}+\Phi \mathbf{A}^{-1} \Phi^{T}$.

Reference [28] proposed fast marginal likelihood maximization for sparse Bayesian models, which can reduce the learning time of RVM effectively. To simplify forthcoming expressions, it is defined that

$$
\begin{aligned}
s_{i} & =\phi_{i}^{T} \mathbf{C}_{-i}^{-1} \phi_{i}, \\
q_{i} & =\phi_{i}^{T} \mathbf{C}_{-i}^{-1} \mathbf{t} .
\end{aligned}
$$

It is showed that (20) has a unique maximum with respect to $\alpha_{i}$ :

$$
\begin{aligned}
& \alpha_{i}=\frac{s_{i}^{2}}{q_{i}^{2}-s_{i}}, \quad \text { if } q_{i}^{2}>s_{i}, \\
& \alpha_{i}=\infty, \quad \text { if } q_{i}^{2} \leq s_{i} .
\end{aligned}
$$

3.2.2. Wrong Codes Recognition Using RVM. We use RVM clustering approach to classify the candidate codes into right bits and wrong ones. Firstly, get the output of the matched filters by (6) and (7), the output of the matched filter group is $\mathbf{y}$, and the output of sign detectors is $\mathbf{b}$. Secondly compared with the vector $\mathbf{b}$ and the correct bits vector $\mathbf{d}$, we can classify the candidate codes into right set $m_{1}$ and wrong set $m_{2}$; let $m_{1}$ and $m_{2}$ be the training samples to train the RVM. Thirdly two useful feature parameters of the training samples can be extracted below: $\mathbf{y}$ and $|L(\mathbf{b})|$. Fourthly use the training samples $m_{1}$ and $m_{2}$ to train the RVM. Fifthly use the trained RVM clustering approach to classify the candidate codes into right bits and wrong ones.

\section{Results and Discussions}

In this section, simulation results are presented in order to show the superiority of the hybrid multiuser detector using code mapping based on relevance vector machine with respect to both system performance improvement and computational complexity reduction.

We present simulation results for the performance of a DS-UWB system with four types of multiuser detector: (1) the matched filter detector; (2) the MMSE multiuser detector; (3) the OMD; and (4) the proposed hybrid multiuser detector. Set the chip transmission rate as $2.55 \mathrm{GHz}$ with the UWB transmission bandwidth $4 \sim 8 \mathrm{GHz}$ and use length 255 Kasami spreading codes for the transmitters. Assume that the signal was transmitted in two typical channels: one is AWGN channel without ISI and the other is IEEE 802.15.3a multipath channel and the multipath signal is processed by a selective-RAKE (SRAKE) receiver with 8 branches and using MRC (Maximal Ratio Combining) rule. Our main objective was to examine a receiver's BER which directly bases the performance of the receiver on the condition of different SNR environments. In addition, we can check the recognition rate to identify the SNR in this scenario.
4.1. Results of Experiment 1: BER Performance versus SNR. First, consider the AWGN channel environment and IEEE 802.15.3a channel model (without loss of generality, CM2 channel is studied in the simulation experiment). There are four kinds of detectors to be simulated: matched filter detector, MMSE detector, OMD, and the proposed hybrid multiuser detector. The number of transmitters is set to 10 , the spread spectrum gain $N_{c}$ equals 255 , and the range of SNR output from the receiver is $0 \mathrm{~dB} \sim 12 \mathrm{~dB}$. The curves of BER performance versus SNR are depicted in Figure 3.

As shown in Figure 3, on the whole, the BER performance of the hybrid multiuser detector is superior to that of the matched filter detector and the MMSE detector in both AWGN channel and the IEEE 802.15.3a multipath CM2 channel. Especially in conditions of high SNR (greater than $8 \mathrm{~dB}$ ), the detection performance of the hybrid multiuser detector is much better than that of the other two detectors (approximately 1 3 orders of magnitude higher). Hence, what is more important is that the BER performance of the hybrid multiuser detector is very close to that of OMD. In theory, the OMD can completely eliminate the MAI and can arrive at the lower limit of BER in the condition of multiple users. This means that the hybrid multiuser detector can correct almost all the wrong codes caused by MAI. Another experiment was carried out to study the BER performance of the proposed hybrid MUD method in different IEEE 802.15.3a channel models, which is presented in Figure 4. Seen from Figure 4, the proposed method is sensitive to channel environments; the performance trend worsened with the deterioration of the channel environment.

4.2. Results of Experiment 2: User Capacity of System. Furthermore, consider another situation with different number of transmitters under the same SNR condition. To make the comparison apparent, the algorithms of matched filter detector MMSE and hybrid multiuser detector are simulated. The simulation results are shown in Figure 5 with the SNR of $4 \mathrm{~dB}$, $6 \mathrm{~dB}, 8 \mathrm{~dB}$, and $10 \mathrm{~dB}$; the other simulation environments yield the same results as BER performance.

Based on the results in Figure 5, it can be seen that the BER performance of the hybrid multiuser detector remains nearly unchanged with an increase in the number of transmitters compared with that of the matched filter receiver. This shows that the hybrid multiuser detection can suppress multiaccess interference. Therefore, under the stated specific SNR conditions, the hybrid multiuser detector is still able to give a superior detection performance when there are many transmitters occupying the same channel simultaneously, which indicates that the BER performance of the hybrid multiuser detector is not sensitive to the channel capacity. This is one of the great advantages of this type of detector.

4.3. Results of Experiment 3: The Near-Far Effect (NFE) Resistant Capability. Due to the spatial distribution of terminals, NFE is one of the outcomes in Multiple Address (MA) systems, which is discussed in this simulation in both AWGN channel and IEEE 802.15.3a CM2 channel. In this experiment, the number of users was set as 10; the first user received 


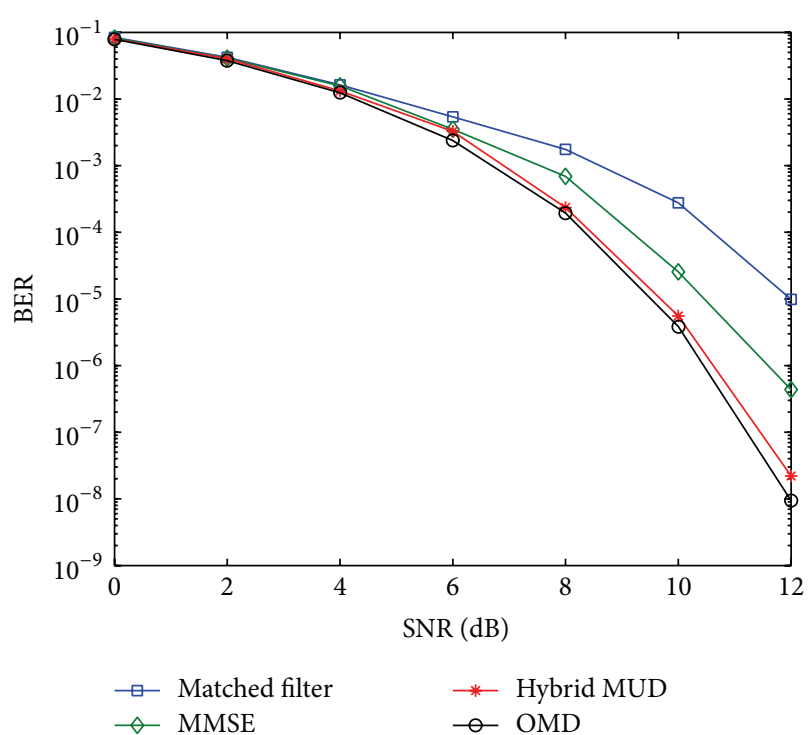

(a)

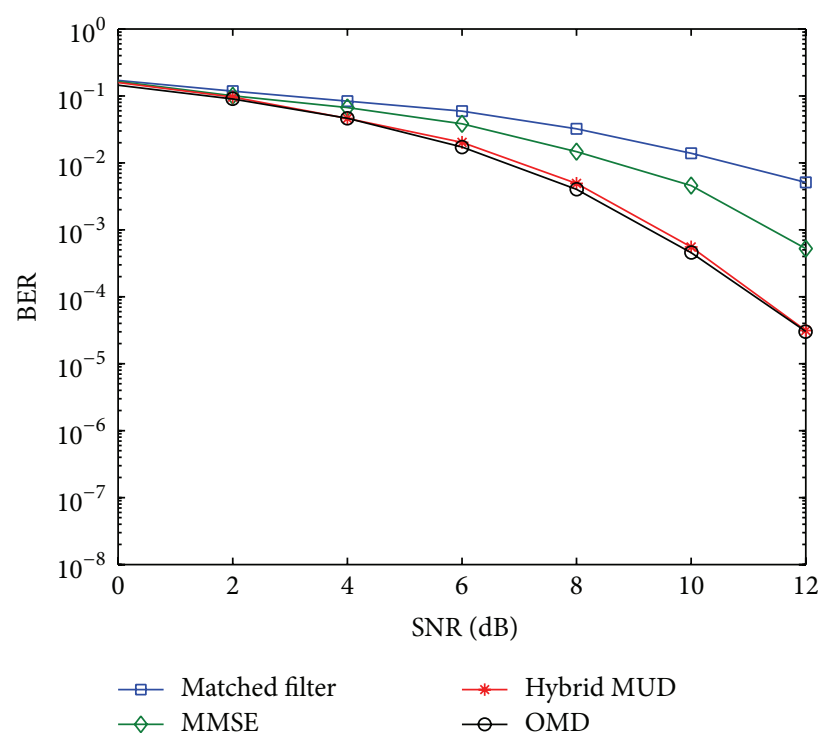

(b)

FIGURE 3: (a) BER performance versus SNR in 10-user condition for AWGN channel. (b) BER performance versus SNR in 10-user condition for multipath CM2 channel.

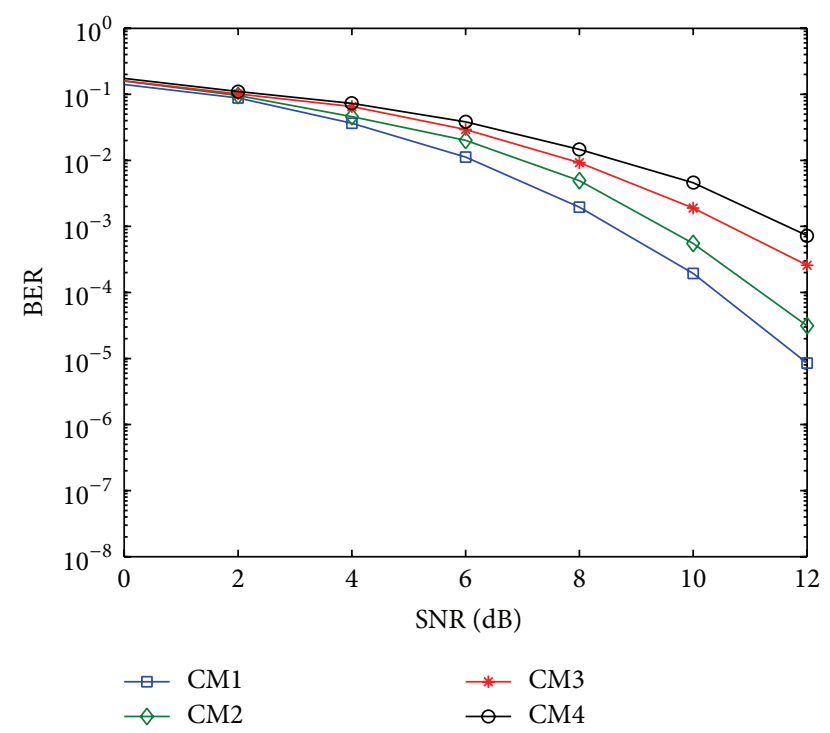

FIGURE 4: BER performance of the proposed method in 4 IEEE 802.15.3a channels.

signal with the stationary SNR of $6 \mathrm{~dB}$, while in that of other users, SNR varies from 0 to $12 \mathrm{~dB}$ synchronously. And other parameters are the same as those in the former experiments.

The near-far effect resistant abilities of different multiuser detector in both AWGN channel and IEEE 802.15.3a CM2 channel are shown in Figure 6. It is evident that the matched filter detector has the worst NFE resistance ability, and both the proposed hybrid multiuser detector and the MMSE have the stronger NFE resistant ability compared to matched filter detector. However, considering the BER performance of them, hybrid MUD is the best.
TABLE 1: The relative execution time of different multiuser detectors.

\begin{tabular}{lcccc}
\hline $\begin{array}{l}\text { Multiuser } \\
\text { detectors }\end{array}$ & Matched filter & MMSE & OMD & Hybrid MUD \\
\hline $\begin{array}{l}\text { Relative } \\
\text { execution time }\end{array}$ & 1 & 1.41 & 58.7 & 1.26 \\
\hline
\end{tabular}

4.4. Results of Experiment 4: Computational Complexity. $\mathrm{OMD}$ can obtain the best BER performance. However, as is shown in (8), in OMD the value of $\left(2 \mathbf{b}^{T} \mathbf{A y}-\mathbf{b}^{T} \mathbf{A R A b}\right)$ needs $2^{K}$ computations to find the maximum one ( $K$ is the number of users). The multiuser proposed in this paper computes the mapping function $\left|L\left(b_{k}\right)\right|$ and employs RVM to distinguish the wrong codes and then correct them. Let the execution time of matched filter normalize to 1 . In an environment of 10user AWGN channel, Table 1 lists the relative execution time of matched filter, MMSE, OMD, and the proposed hybrid MUD.

From Table 1, it can be seen that the computational complexity of the proposed hybrid multiuser detector is much lower than that of OMD. And it is in the same order of magnitude to matched filter and MMSE. Thus the proposed multiuser detector based on code mapping and RVM can get good BER performance with computational complexity.

\section{Conclusions}

In this paper, a new MAI suppression mechanism is proposed for multiuser detection of DS-UWB system. The algorithm embraces three steps. First, as the output of matched filters, the candidate codes set is mapped into a one-dimensional feature space by the optimal decision function. The purpose is to make the right codes and wrong codes easy to be 


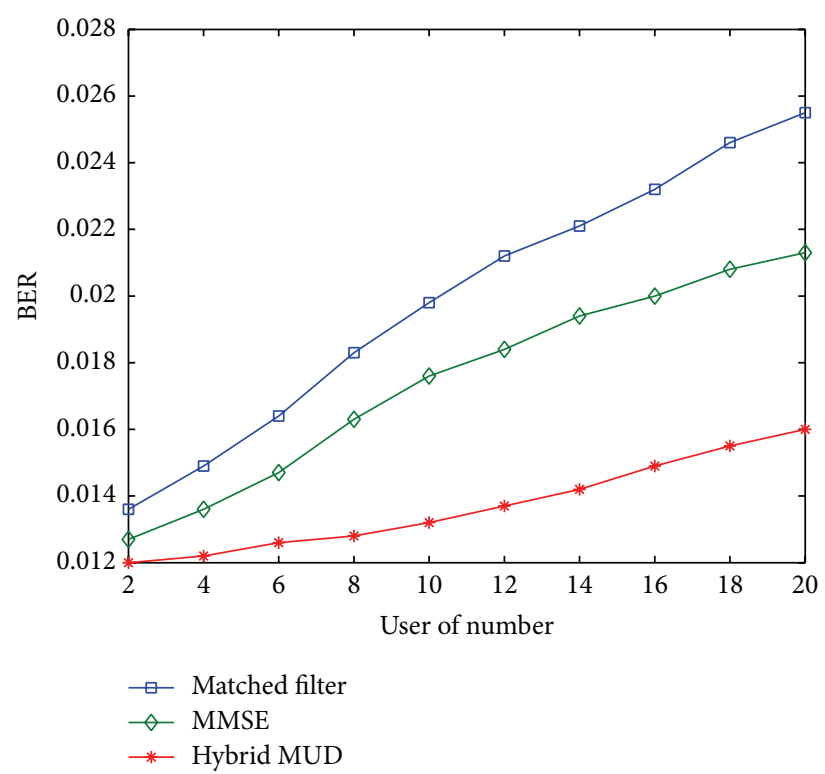

(a)

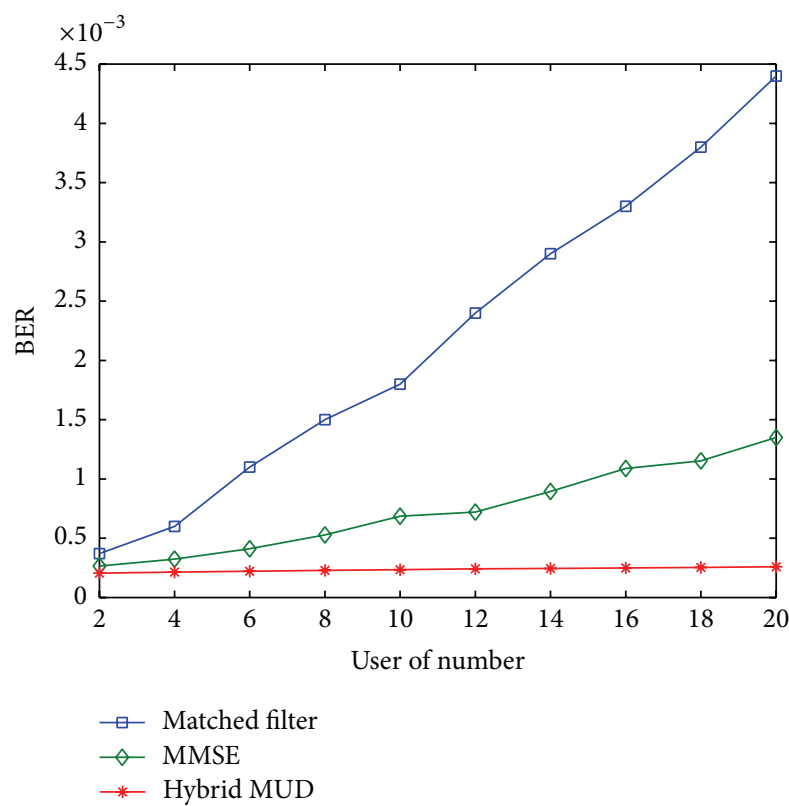

(c)

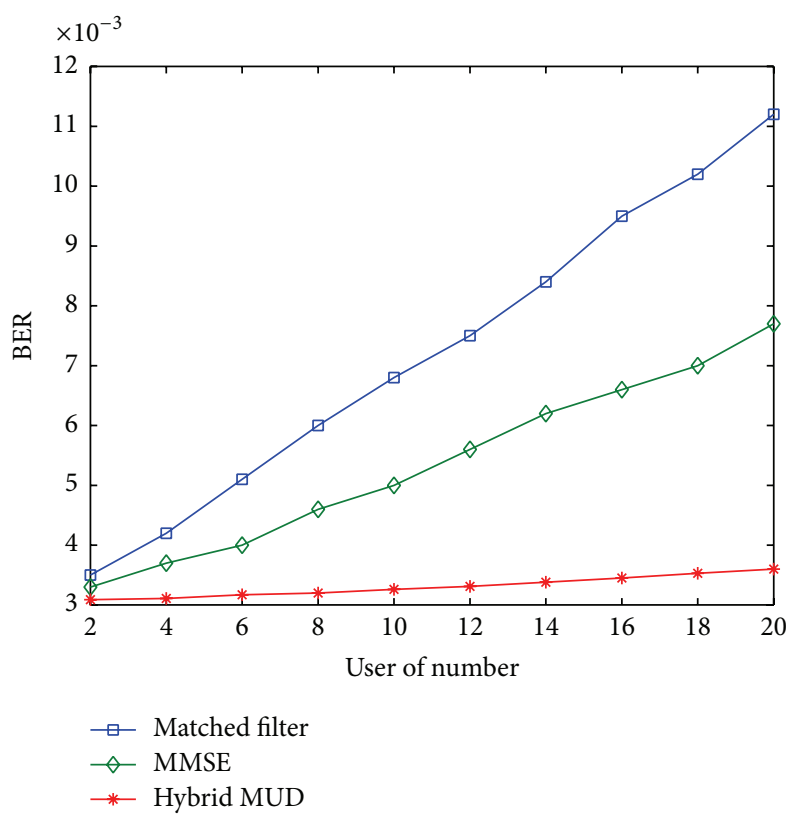

(b)

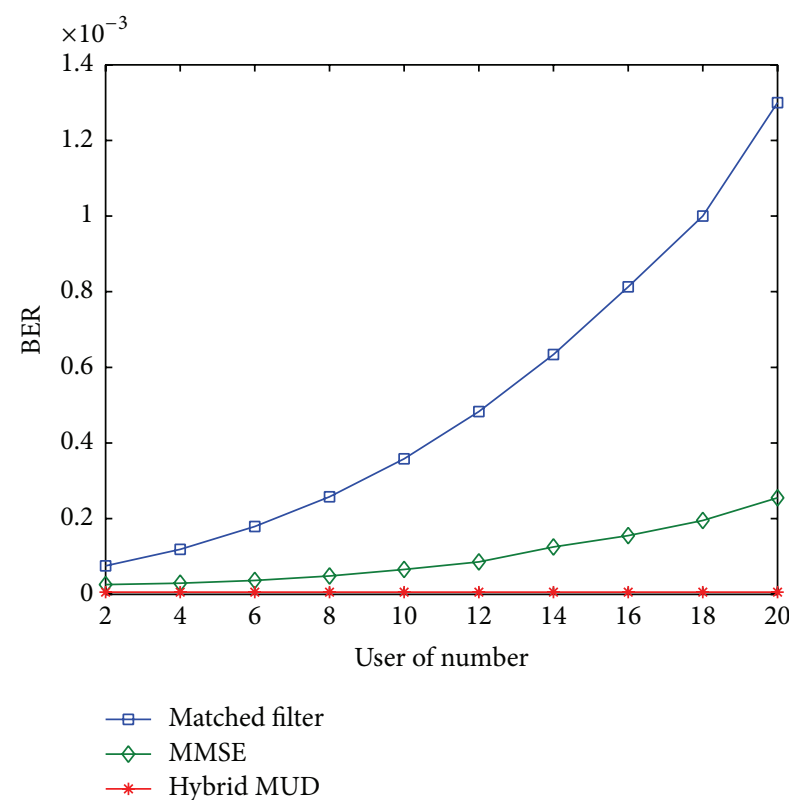

(d)

FIGURE 5: BER performance versus the number of users in specific SNR condition. (a) $\mathrm{SNR}=4 \mathrm{~dB}$, (b) $\mathrm{SNR}=6 \mathrm{~dB},(\mathrm{c}) \mathrm{SNR}=8 \mathrm{~dB}$, and (d) $\mathrm{SNR}=10 \mathrm{~dB}$.

distinguished. Second, calculate the statistical characteristics of the codes in the feature space. Third, use RVM classifier to classify the receiver multiuser function mapping characteristic value, and then correct the wrong codes by sign reversal. The simulation results show that the BER performance and the near-far effect resistant abilities of the new MAI suppression mechanism are superior to those of the matched filter detector and the MMSE detector in both AWGN channel and IEEE 802.15.3a channel, especially when the output SNR is greater than $8 \mathrm{~dB}$. Its performance is very close to that of OMD. Compared to the OMD, the new MAI suppression mechanism has lower computational complexity that increases linearly with the number of users. Hence, we conclude that the new MAI suppression mechanism can give an admirable detection performance under high SNR and large number of users, which is very useful for practical applications. Furthermore, the performance of the new MAI suppression mechanism can hardly be influenced by changes in numbers of users, which means that this hybrid approach for multiuser detection will be an efficient method to solve 


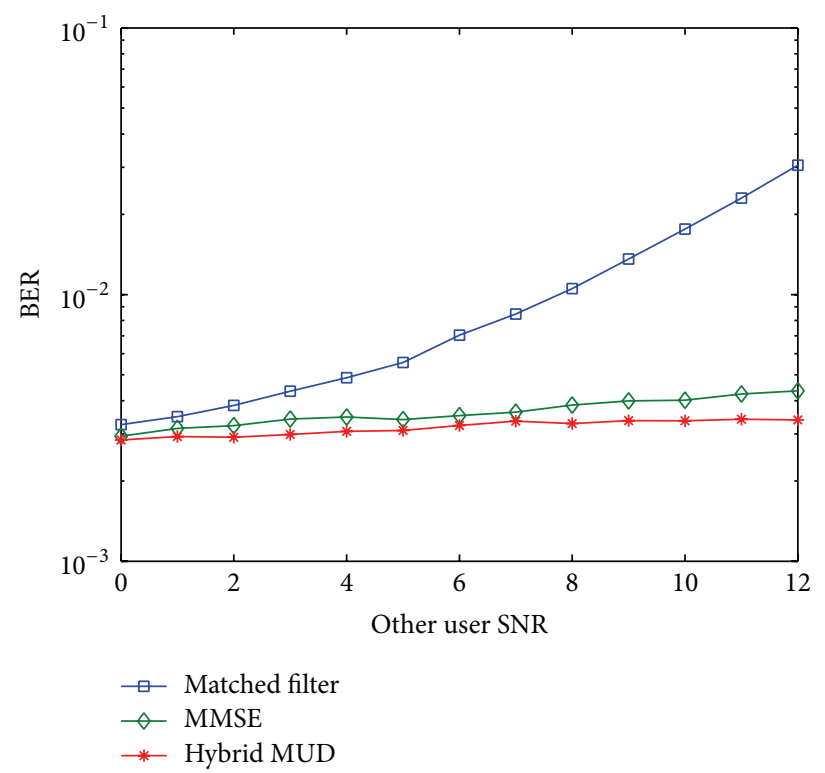

(a)

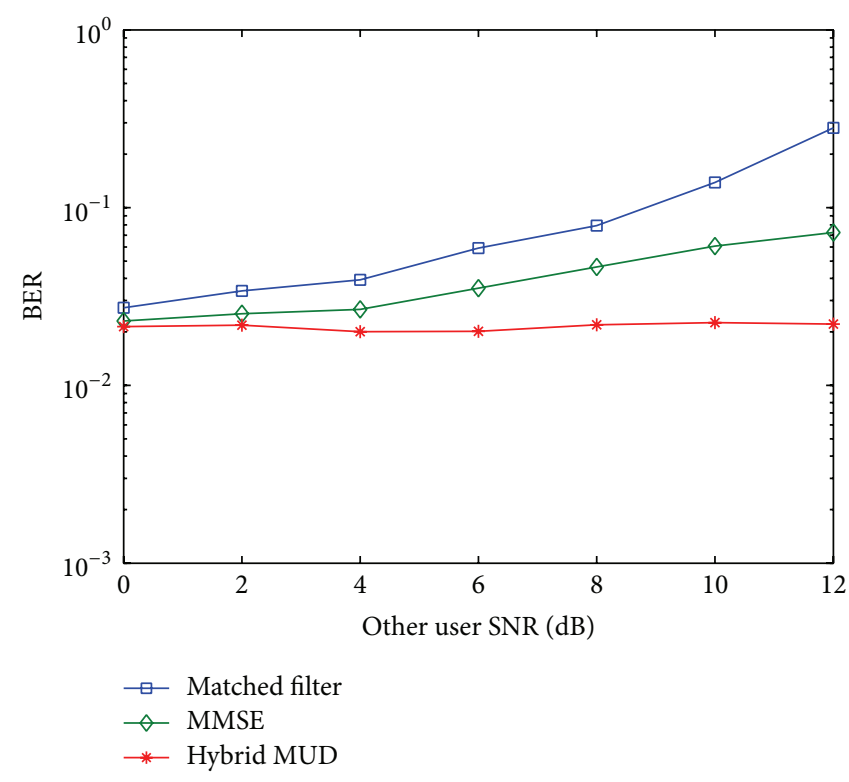

(b)

FIGURE 6: (a) The near-far effect resistant abilities of different MUD methods in AWGN channel. (b) The near-far effect resistant abilities of different MUD methods in CM2 channel.

interference problems when there are large quantities of transmitters occupying the same channel simultaneously.

\section{Conflict of Interests}

The authors declare that there is no conflict of interests regarding the publication of this paper.

\section{Acknowledgments}

The research in this paper is supported by the National Natural Science Foundation of China (Grant no. 61102084), the Fundamental Research Funds for the Central Universities in China (Grant no. HIT.NSRIF.2010092), and the China Postdoctoral Science Foundation (Grant no. 2011M500665).

\section{References}

[1] S. J. Ahmadi and R. C. Qiu, "Ultra-wideband (UWB) communications channel measurements-a tutorial review," International Journal of Ultra Wideband Communications and Systems, vol. 1, no. 1, pp. 11-30, 2009.

[2] X. Shen, M. Guizani, H. H. Chen, R. C. Qiu, A. F. Molisch, and L. B. Milstein, "Guest editorial ultra-wideband wireless communications-theory and applications," IEEE Journal on Selected Areas in Communications, vol. 24, no. 4, pp. 713-716, 2006.

[3] H. Zhang, S. Hong, and K. Chang, "Class 4 active RFID multihop relay system based on IEEE 802.15.4a low-rate UWB in sensor network," KSII Transactions on Internet and Information Systems, vol. 4, no. 3, pp. 258-272, 2010.

[4] Z. L. Xiong and Q. S. Xiang, "A novel multi-user detector in UWB communication systems," in Proceedings of the Sensor
Array and Multichannel Signal Processing Workshop Proceedings, pp. 129-132, IEEE, Barcelona, Spain, July 2004.

[5] J. Chóliz, Á. Hernández, and A. Valdovinos, "A framework for UWB-based communication and location tracking systems for wireless sensor networks," Sensors, vol. 11, no. 9, pp. 9045-9068, 2011.

[6] M. H. Kabir and R. Kohno, "A hybrid TOA-fingerprinting based localization of mobile nodes using UWB signaling for non lineof-sight conditions," Sensors, vol. 12, no. 8, pp. 11187-11204, 2012.

[7] J. Maunu, T. Koivisto, M. Laiho, and A. Paasio, "An analog viterbi decoder array for DS-UWB receiver," in Proceedings of the IEEE International Conference on Ultra-Wideband (ICUWB '06), pp. 31-36, Waltham, Mass, USA, September 2006.

[8] S.-S. Tan, A. Nallanathan, and B. Kannan, "Performance of DS-UWB multiple-access systems with diversity reception in dense multipath environments," IEEE Transactions on Vehicular Technology, vol. 55, no. 4, pp. 1269-1280, 2006.

[9] H. Sato and T. Ohtsuki, "Frequency domain channel estimation and equalization for direct sequence ultra wideband (DS-UWB) system," IEE Proceedings: Communications, vol. 153, no. 1, pp. 93-98, 2006.

[10] S. Verdu, "Optimum multiuser asymptotic efficiency," IEEE Transactions on Communications, vol. 34, no. 9, pp. 890-897, 1986.

[11] Y. C. Yoon and R. Kohno, "Optimum multi-user detection in ultra-wideband (UWB) multiple-access communication systems," in Proceedings of the International Conference on Communications (ICC '02), pp. 812-816, New York, NY, USA, May 2002.

[12] Z. Yin, X. Liu, and Z. Wu, "A multiuser detector based on artificial bee colony algorithm for ds-uwb systems," The Scientific World Journal, vol. 2013, Article ID 547656, 8 pages, 2013.

[13] Z. Yin, Z. Zong, H. Sun, Z. Wu, and Z. Yang, "A complexityperformance-balanced multiuser detector based on artificial 
fish swarm algorithm for DS-UWB systems in the AWGN and multipath environments," EURASIP Journal on Advances in Signal Processing, vol. 2012, no. 1, article 229, 13 pages, 2012.

[14] P. Kaligineedi and V. K. Bhargava, "Frequency-domain turbo equalization and multiuser detection for DS-UWB systems," IEEE Transactions on Wireless Communications, vol. 7, no. 9, pp. 3280-3284, 2008.

[15] C. Wang, M. Ma, R. Ying, and Y. Yang, "Narrowband interference mitigation in DS-UWB systems," IEEE Signal Processing Letters, vol. 17, no. 5, pp. 429-432, 2010.

[16] G. S. Biradar, S. N. Merchant, and U. B. Desai, "Performance of constrained blind adaptive DS-CDMA UWB multiuser detector in multipath channel with narrowband interference," in Proceedings of the IEEE Global Telecommunications Conference (GLOBECOM '08), pp. 1-5, IEEE, New Orleans, La, USA, December 2008.

[17] Q. Z. Ahmed and L.-L. Yang, "Reduced-rank adaptive multiuser detection in hybrid direct-sequence time-hopping ultrawide bandwidth systems," IEEE Transactions on Wireless Communications, vol. 9, no. 1, pp. 156-167, 2010.

[18] X. Wang and H. Vincent Poor, "Iterative (Turbo) soft interference cancellation and decoding for coded CDMA," IEEE Transactions on Communications, vol. 47, no. 7, pp. 1046-1061, 1999.

[19] R. M. Cramer, M. Z. Win, and R. A. Scholtz, "Impulse radio multipath characteristics and diversity reception," in Proceedings of IEEE International Conference on Communications (ICC '98), pp. 1650-1654, Atlanta, Ga, USA, June 1998.

[20] C. Thejaswi, P. S. Manohar, S. V. Ganapathy, R. Patro, and M. Raina, "Simple multiuser detectors for DS-UWB systems," in Proceedings of the 17th Annual IEEE International Symposium on Personal, Indoor and Mobile Radio Communications, pp. 1-5, IEEE, Helsinki, Finland, September 2006.

[21] Z. Yin, Y. Kuang, Z. Wu, and W. Tang, "A hybrid multiuser detector for DS-UWB systems with matched filter and errorbit recognizer," Journal of the Chinese Institute of Engineers, vol. 35, no. 8, pp. 1003-1012, 2012.

[22] S. Verdu, "Minimum probability of error for asynchronous Gaussian multiple-access channels," IEEE Transactions on Information Theory, vol. 32, no. 1, pp. 85-96, 1986.

[23] M. E. Tipping, "Sparse Bayesian learning and the relevance vector machine," Journal of Machine Learning Research, vol. 1, no. 3, pp. 211-244, 2001.

[24] M. E. Tipping, "Fast marginal likelihood maximisation for sparse bayesian models," in Proceedings of the 9th International Workshop on Artificial Intelligence and Statistics, Key West, Fla, USA, January 2003.

[25] P.-K. Wong, Q. Xu, C.-M. Vong, and H.-C. Wong, "Ratedependent hysteresis modeling and control of a piezostage using online support vector machine and relevance vector machine," IEEE Transactions on Industrial Electronics, vol. 59, no. 4, pp. 1988-2001, 2012.

[26] Q. Xu and P.-K. Wong, "Hysteresis modeling and compensation of a piezostage using least squares support vector machines," Mechatronics, vol. 21, no. 7, pp. 1239-1251, 2011.

[27] Z. Yang, Z. Wu, Z. Yin, T. Quan, and H. Sun, "Hybrid radar emitter recognition based on rough k-means classifier and relevance vector machine," Sensors, vol. 13, no. 1, pp. 848-864, 2013.

[28] E. E. Azzouz and A. K. Nandi, "Automatic identification of digital modulation types," Signal Processing, vol. 47, no. 1, pp. 55-69, 1995. 


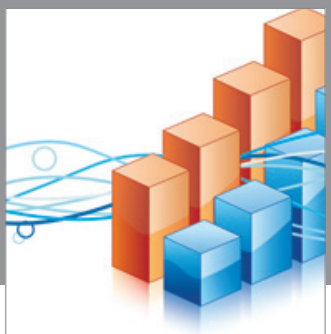

Advances in

Operations Research

vatem alat4

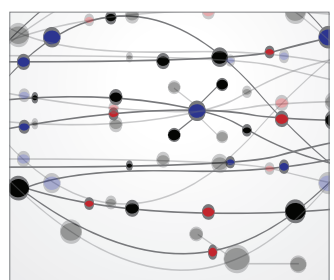

\section{The Scientific} World Journal
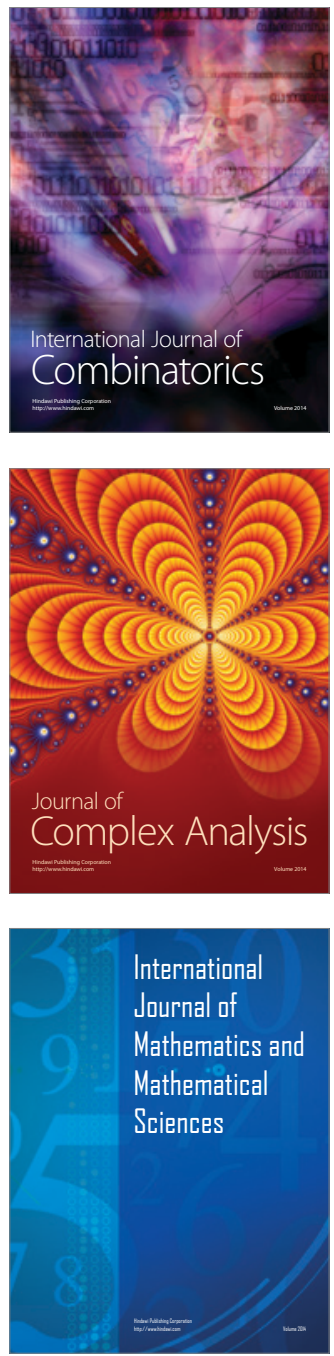
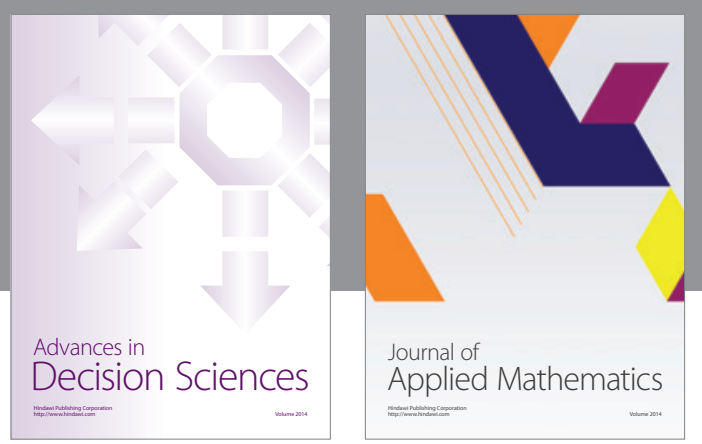

Algebra

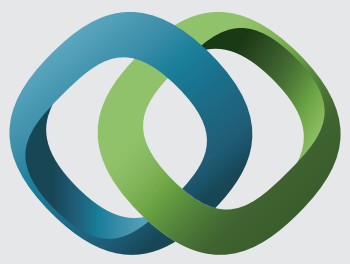

\section{Hindawi}

Submit your manuscripts at

http://www.hindawi.com
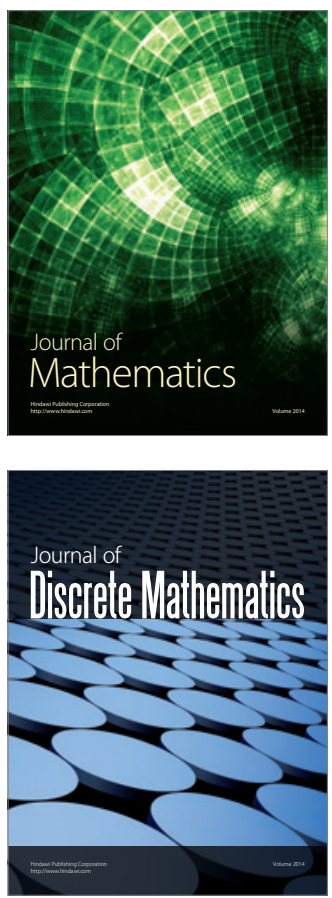

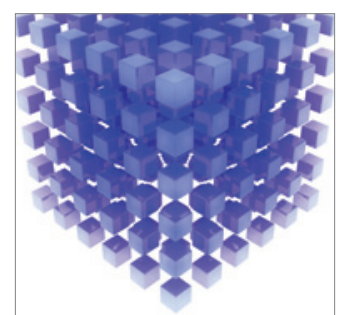

Mathematical Problems in Engineering
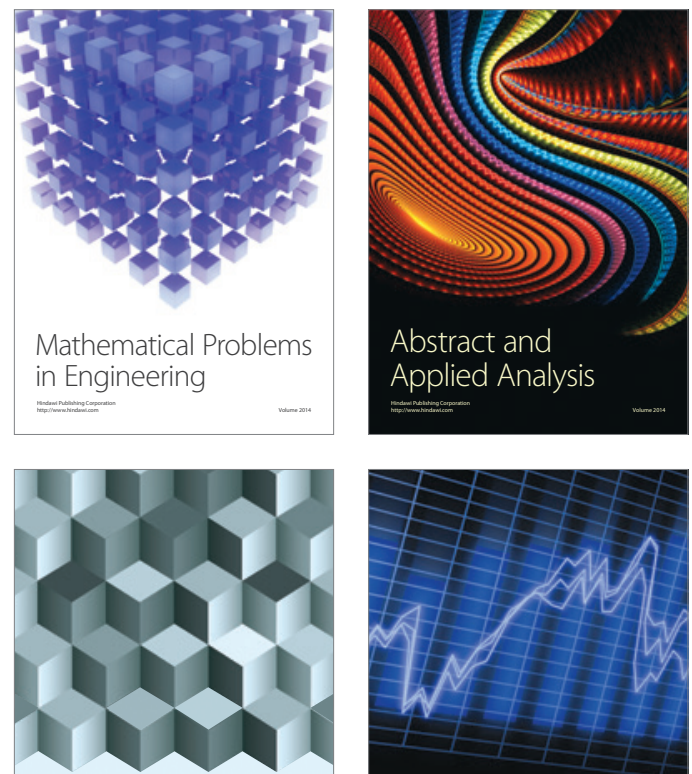

Journal of

Function Spaces

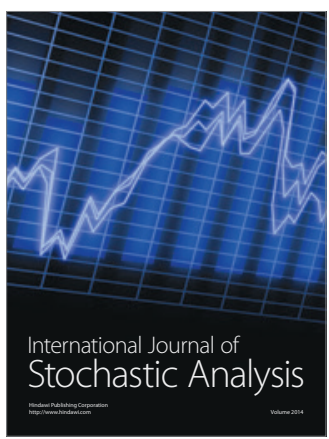

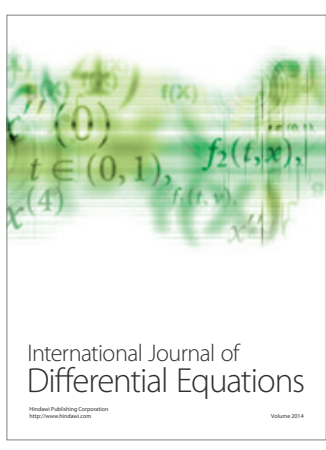
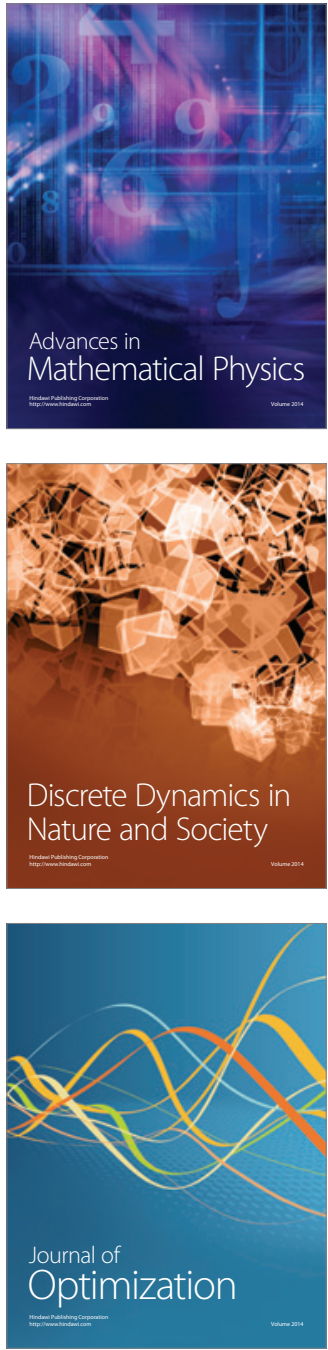